Research Paper

\title{
Silencing of Survivin Expression Leads to Reduced Pro- liferation and Cell Cycle Arrest in Cancer Cells
}

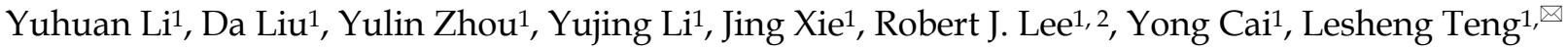 \\ 1. Institute of Life Sciences, Jilin University, Changchun, Jilin, P. R. China \\ 2. Division of Pharmaceutics, College of Pharmacy, The Ohio State University, Columbus, OH, U.S.A \\ $\triangle$ Corresponding author: Lesheng Teng, PhD, Associate Professor, Institute of Life Sciences, Jilin University, Changchun, Jilin, P. R. China, Email: \\ tenglesheng@jlu.edu.cn; TEL: +86 431 85168646; FAX: +86 43185168637
}

() 2015 Ivyspring International Publisher. Reproduction is permitted for personal, noncommercial use, provided that the article is in whole, unmodified, and properly cited. See http://ivyspring.com/terms for terms and conditions.

Received: 2015.04.17; Accepted: 2015.07.27; Published: 2015.09.15

\begin{abstract}
Survivin is an anti-apoptotic gene that is overexpressed in most human tumors. RNA interference using short interfering RNA (siRNA) can be used to specifically inhibit survivin expression. Tumor cells were treated with a newly designed survivin siRNA, which was modified with 2'-OMe. Cellular survivin mRNA and protein levels were determined by real-time qRT-PCR and Western blot, respectively. Cell cycle and apoptosis were determined by flow cytometry. Cell proliferation was measured by MTT assay. Our data showed that the novel survivin-targeted siRNA could efficiently knockdown the expression of survivin and inhibit cell proliferation. Survivin mRNA was reduced by $95 \%$ after $48 \mathrm{~h}$ treatment with $20 \mathrm{nM}$ siRNA. In addition, the siRNA could markedly arrest the cell cycle at the G2/M checkpoint and induce cellular apoptosis in a dose-dependent manner. The percentage of apoptotic cells reached $50 \%$ when treated with $40 \mathrm{nM}$ siRNA. In conclusion, we have identified a novel chemically modified siRNA against survivin that is highly efficient and delineated its mechanism of action, thus demonstrating a potential therapeutic role for this molecule in cancer. Further evaluation of this siRNA for therapeutic activity is warranted.
\end{abstract}

Key words: survivin, RNA interference, cancer, apoptosis, cell cycle checkpoint

\section{Introduction}

Survivin is a member of the inhibitor of apoptosis (IAP) protein family [1, 2]. It inhibits apoptosis and regulates cell division [3-6]. Sustained overexpression of survivin has been shown to be cancer specific [7-9]. In addition, elevated expression of survivin plays a significant role in the inhibition of apoptosis [10-13].These factors suggest that survivin is a potential therapeutic target [14].

Growth inhibition and apoptosis induction are important mechanisms of cancer therapy [15]. RNA interference (RNAi) by small interfering RNA (siRNA) can be used to reduce target gene expression in a sequence specific manner by degradation of the corresponding mRNA [16-19]. After uptake by cells, siRNA is loaded into a RNA-induced silencing complex (RISC) [20, 21]. The passenger strand is then degraded and the remaining strand (guide strand) binds to a complementary RNA molecule, which is then degraded [22]. Gene silencing induced by siRNA is highly efficient and specific to the target gene and therefore has potential application in cancer treatment $[23,24]$.

In recent years, several siRNA sequences targeting survivin have been reported [25]. However, they generally show only moderate activity [26]. Unmodified siRNA have issues such as poor stability, off-target effect and immune stimulation [27]. Indeed, modifications of the siRNA backbone by chemical groups, such as 2'-O-methyl (OMe) and 2'-fluoro (F), alone or in combination [28, 29], can improve serum stability and reduce off-target effects [30]. However, siRNA modification can adversely affect its gene-silencing activity, thus presenting a critical challenge for siRNA drug development [31]. 
In order to achieve maximum therapeutic effect, it is essential to identify the most active form of drugs. Therefore, several 2'-OMe chemical groups were introduced into a novel survivin siRNA (siRNA-1) and the improvement in potency was evaluated in vitro in the present study.

\section{Results and Discussion}

\subsection{Down-regulation of survivin in human tumor cell lines}

Silencing of survivin expression was examined in a number of cell lines representing different types of tumors (MCF-7, A549, HeLa, and HepG2). Following transfection of cells with $10 \mathrm{nM}$ siRNA-1, the protein of survivin was determined by Western blot. HeLa and A549 cells had higher expression of survivin compared with the HepG2 and MCF-7 cells. In these cell lines, the siRNA targeting survivin successfully down-regulated the expression levels of survivin protein after $48 \mathrm{~h}$ treatment with siRNA-1 (Figure 1A). The mRNA levels of survivin were determined by real-time qRT-PCR at $48 \mathrm{~h}$ after transfection with different concentrations of siRNA-1 in HeLa cells. As shown in Figure 1B, survivin transcription was reduced by more than $70 \%$ at the transcriptional level. At 20nM siRNA, survivin mRNA was reduced by $95 \%$. Analysis by immunofluorescence revealed survivin localization in the nucleus. In cells treated with increasing concentrations of siRNA-1, the fluorescence intensity was gradually diminished (Figure 1C). The cells treated with $20 \mathrm{nM}$ siRNA-1 had the

$\mathbf{A}$

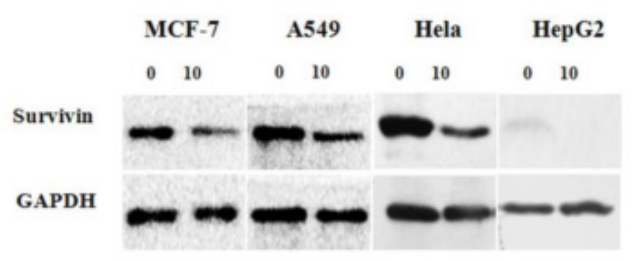

B

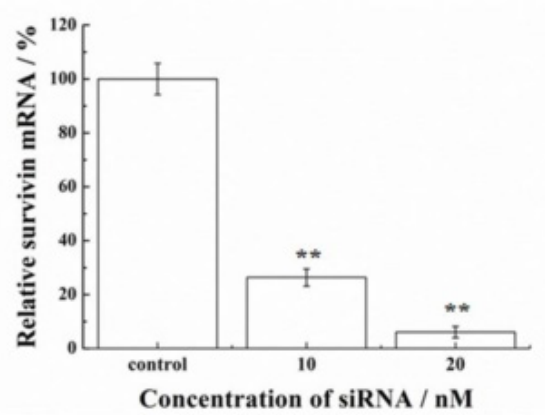

C

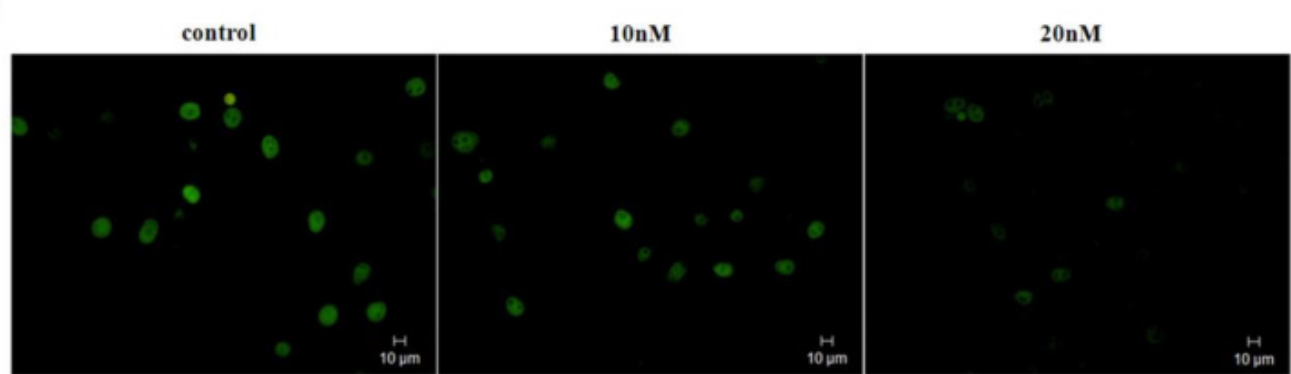

Figure 1. Survivin silencing by siRNA-1 in a number of cell lines. (A) Survivin expression analyzed by Western blot $48 \mathrm{~h}$ after transfection with siRNA-1. (B) The levels of survivin mRNA determined by real-time qRT-PCR $48 \mathrm{~h}$ after transfection in HeLa cells. (C) Survivin expression analyzed by immunofluorescence after transfection with $10 \mathrm{nM}$ or $20 \mathrm{nM}$ siRNA-1. **Statistically significant at $p<0.01$. 
A

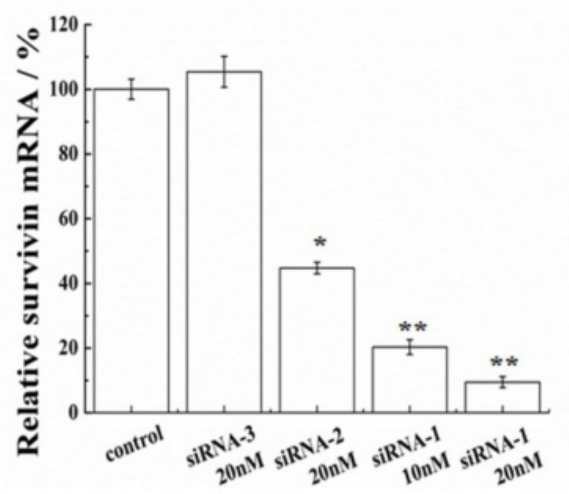

B

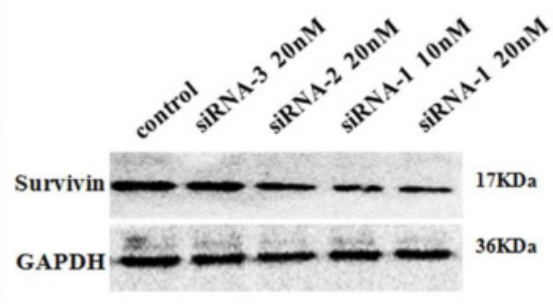

C

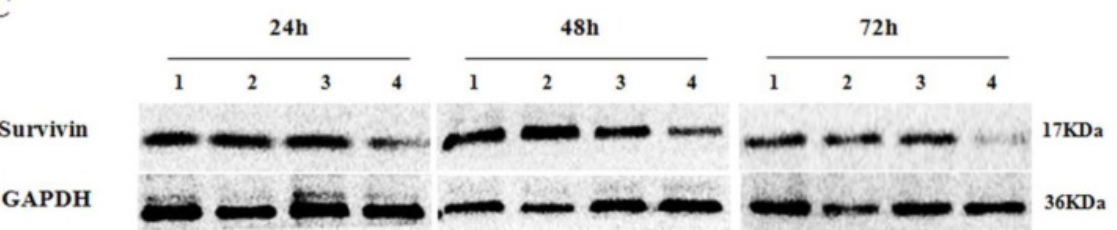

Figure 2. Inhibition of survivin expression in MCF-7 cells. (A) Survivin mRNA levels were analyzed after transfection with siRNAs. Survivin levels were expressed relative to GAPDH. (B) Protein levels of survivin were analyzed by Western blot $48 \mathrm{~h}$ after transfection with siRNAs. (C) Protein levels of survivin were analyzed by Western blot at 24 , 48 and $72 \mathrm{~h}$ after transfection, (1) untreated cells, (2) 20nM siRNA-2, (3) 10nM siRNA-1, (4) 20nM siRNA-1. *Statistically significant with $p<0.05$, **Statistically significant with $p<0.01$.

\section{3. siRNA induced G2/M Cell Cycle Arrest}

In addition to the suppression of survivin, we evaluated the effect of siRNA on proliferation and progression of cell cycle in HeLa cells. Treatment of cells by siRNA- 1 at $48 \mathrm{~h}$ induced $\mathrm{G} 2 / \mathrm{M}$ cell cycle arrest. In addition, the G0/G1 phase ratio decreased significantly (Figure 3A, B). Cell cycle arrest reached the maximum at $48 \mathrm{~h}$ (Figure 3C, D). At $72 \mathrm{~h}$ after transfection, the G2 phase was still blocked. In the meantime, the percentage of cells in the $S$ phase was increased. Based on these data, cell cycle arrest was induced by siRNA-1, mainly at the G2/M phases.

\subsection{Inhibitory effect on cell proliferation by SiRNA}

Treatment of HeLa cells with siRNA-1 over $72 \mathrm{~h}$ caused remarkable reductions in cell proliferation compared to non-treated cells (Figure. 4A). At $72 \mathrm{~h}$ after transfection, cell proliferation inhibition rate reached to nearly $50 \%$. Furthermore, the effect of siRNA-1 on growth of HeLa cells was investigated (Figure. 4B). The number of surviving cells treated with $40 \mathrm{nM}$ siRNA-1 on the fourth day was $50 \%$ relative to the control group.

\section{5. siRNA induces cell apoptosis and alters apoptosis-related signaling molecules in HeLa cells}

The effect on the cell cycle and proliferation from siRNA-1 was obvious. Cell apoptosis was also studied. Treatment of HeLa cells with different concentra- tions of siRNA-1 induced different degrees of apoptosis detected by flow cytometry. As shown in Figure $5 \mathrm{~A}$, the percentage of apoptotic cells increased with the concentration of siRNA- 1 . When the concentration of siRNA reached $40 \mathrm{nM}$, the percentage of apoptotic cells was reached $50 \%$. To characterize the molecular mechanism by which survivin alters apoptosis in HeLa cells, we examined the expression of apoptosis-associated proteins in response to survivin. The expression levels of p-Akt, Bax, and Bad were examined using Western blot at $48 \mathrm{~h}$ after transfection (Figure. 5B). The protein levels were quantified by densitometry (Figure. 5C). Apoptosis-inducing proteins Bax and Bad were increased in response to the down-regulation of survivin. This showed that siRNA-1 promoted cell apoptosis via Bad and Bax. At the same time, Akt signaling pathway was involved, and the level of p-Akt was similarly down-regulated with a decrease of survivin. In a subsequent experiment, we examined whether siRNA could inhibit the decrease of mitochondrial membrane potential $(\Delta \psi \mathrm{m})$ induced by siRNA (Fig.6A). Under the control conditions, JC-1 emitted high intensity of red fluorescence, and with the increasing concentration of siRNA-1, red fluorescence intensity became weaker compared with control. The data demonstrated that siRNA promoted the dissipation of $\Delta \psi \mathrm{m}$, which confirmed that cell apoptosis was induced by the Akt signal pathway. In summary, siRNA-1 can affect cell cycle progression, cause G2/M phase arrest and induce tumor cell apoptosis, particularly through the AKT pathway. 


\section{Experimental Section}

\section{1. siRNA Design}

siRNAs were purchased from Guangzhou RiboBio Co. (Guangzhou, China). The sequences used are as follows (Table 1). siRNA purification was conducted by reverse-phase high performance liquid chromatography (HPLC). The second siRNA (siRNA-2), adopted from a previous study, was used as the positive control targeting survivin [26]. The third siRNA (siRNA-3) was used as a negative control.

A
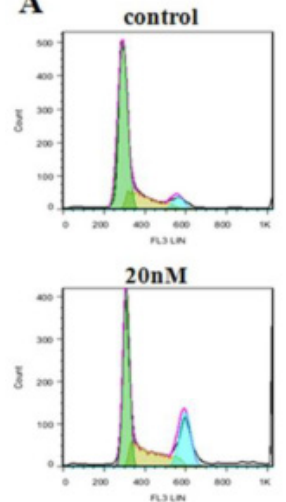

C
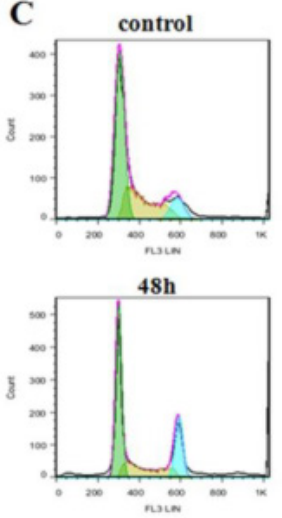
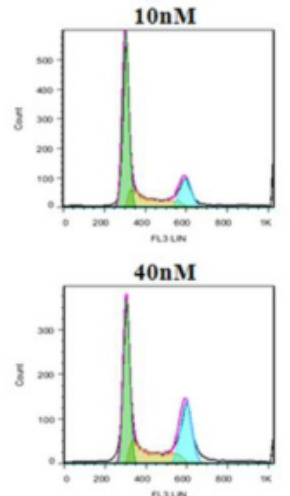

$24 \mathrm{~h}$

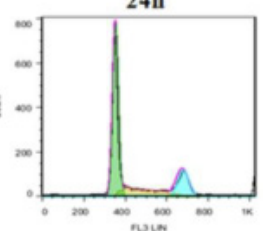

$72 \mathrm{~h}$

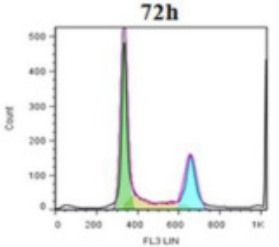

Table 1. siRNAs sequences

\begin{tabular}{|c|c|c|}
\hline \multirow[t]{2}{*}{ siRNA-1 } & sense & mGCA GGU UCC UmUA UCU GUCA dTdT \\
\hline & antisense & UGA mCAG AmUA AGG AAC CUGmCdTdT \\
\hline \multirow[t]{2}{*}{ siRNA-2 } & sense & GGC UGG CUU CAU CCA CUGC dTdT \\
\hline & antisense & GCA GUG GAU GAA GCC AGCC dTdT \\
\hline \multirow[t]{2}{*}{ siRNA-3 } & sense & UUC UCC GAA CGU GUC ACG UTT \\
\hline & antisense & ACG UGA CAC GUU CGG AGA ATT \\
\hline
\end{tabular}

$\mathrm{m}$, represents as a single 2'-OMe modification was made at the position of the siRNA strand.

B

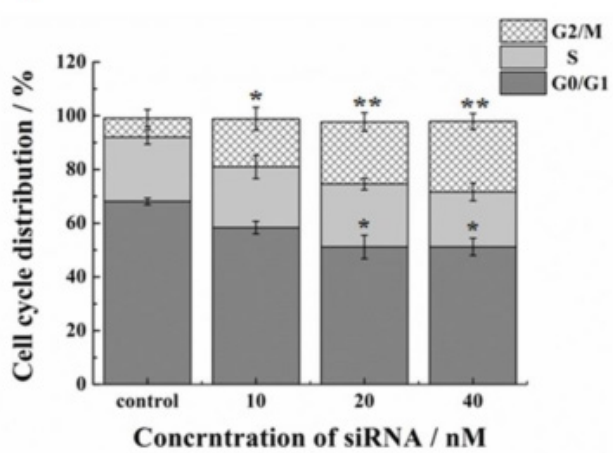

D

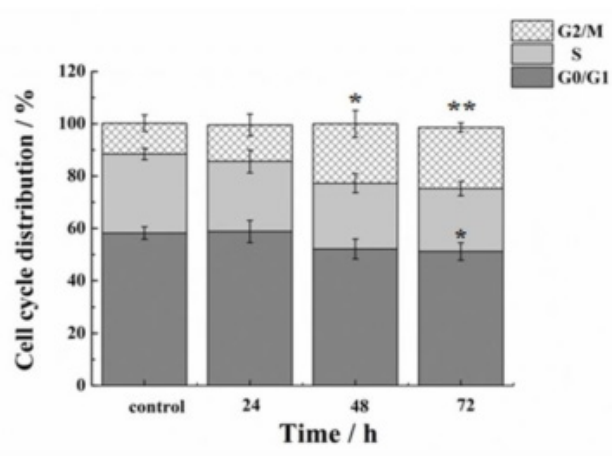

Figure 3. Effects of down-regulation of survivin on cell cycle. (A) Flow cytometry analysis of the cell cycle of HeLa cells at 48h after transfection with different concentrations of siRNA-1. (B) Cell cycle distribution of HeLa cells at $48 \mathrm{~h}$ after transfection with different concentrations of siRNA-1. (C) Flow cytometry analysis of the cell cycle of HeLa cells at 24,48 , and $72 \mathrm{~h}$ after transfection with $40 \mathrm{nM}$ siRNA-1. (D) Cell cycle distribution of HeLa cells at 24,48 , and 72 h following transfection with $40 \mathrm{nM}$ siRNA-1. *Statistically significant with $p<0.05$, **Statistically significant with $p<0.01$.

A

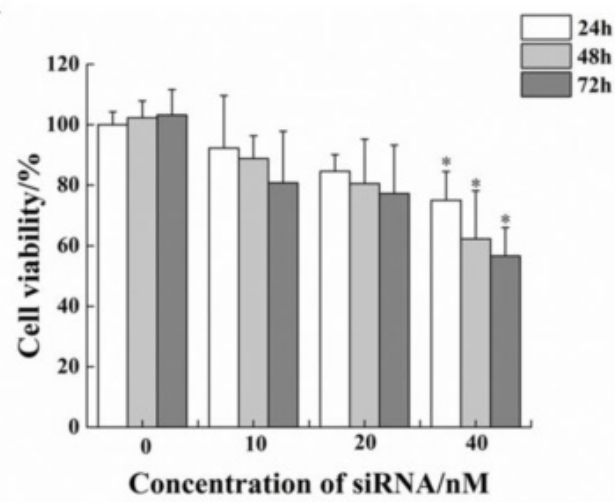

B

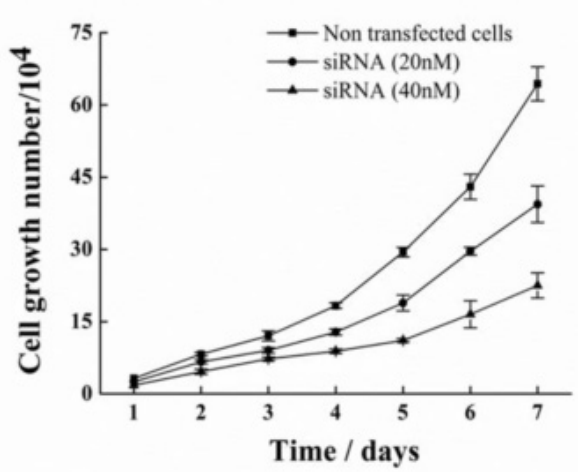

Figure 4. Inhibitory effect on cell proliferation by siRNA-1 targeting survivin. (A) MTT assay analysis of viability of HeLa cells following 24-72 h of treatment with different doses of siRNA-1. (B) Assessment of HeLa cell growth after transfection with different concentrations of siRNA over seven days. *Statistically significant with $p<0.05$. 
A
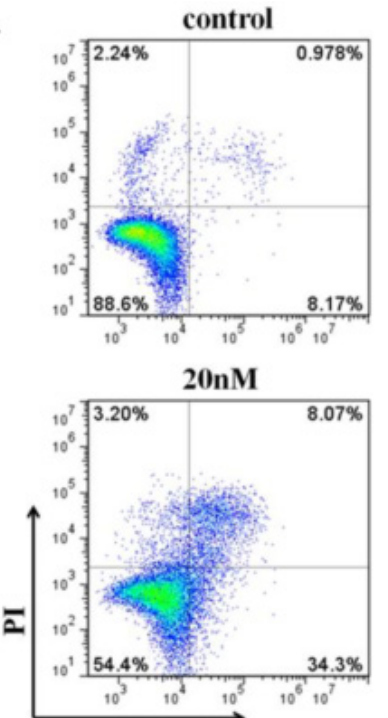

Annexin V-FITC
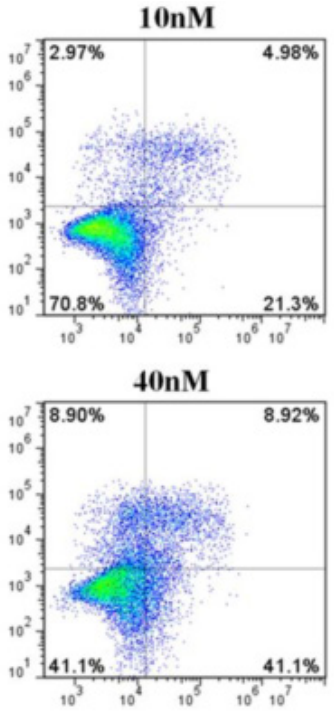

C

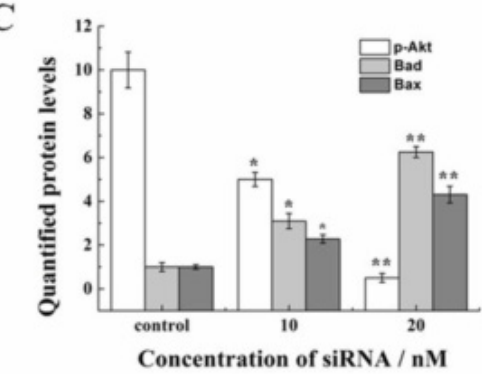

Figure 5.The novel siRNA-1 alters apoptosis-related signaling molecules in HeLa cells. (A) The effect of different concentrations of siRNA-1 on cell apoptosis. (B) The expression levels of the apoptosis signaling proteins Akt, Bax, and Bad examined using Western blot. (C) Quantitation of apoptosis signaling proteins treated by different concentrations of siRNA. *Statistically significant with $p<0.05$, **Statistically significant with $p<0.01$.

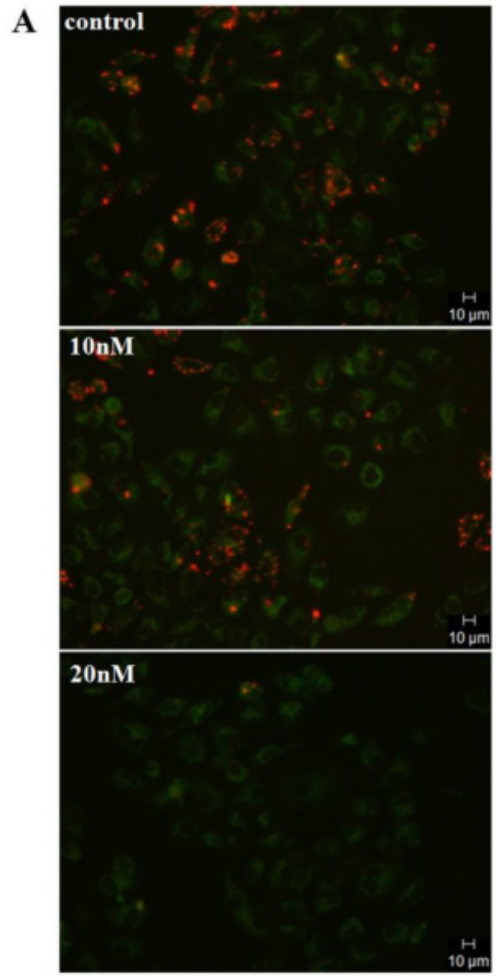

B

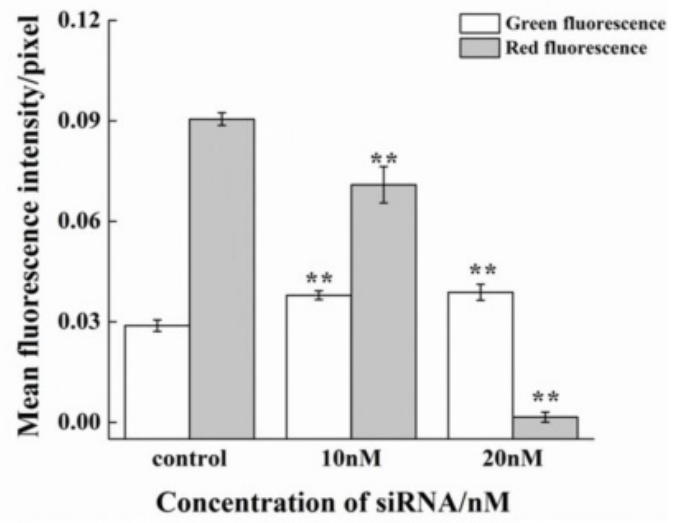

Figure 6. The effect of siRNA-1 on $\Delta \psi \mathrm{m}$ at $48 \mathrm{~h}$ in HeLa cells. (A) HeLa cells exposed to media only (control), 10nM siRNA, or 20nM siRNA. (B) Quantitation of the green and red fluorescence. **Statistically significant with $p<0.01$.

\subsection{Cell lines and cell culture}

Cell lines were purchased from the American Type Culture Collection (ATCC; Rockville, MD). Cells were grown in DMEM or RPMI-1640 medium (Gibco, Grand Island, NY) supplemented with 10\% fetal bovine serum (FBS) and 1\% antibiotics/antimycotics (Sigma-Aldrich, St. Louis, $\mathrm{MO}$ ) at $37^{\circ} \mathrm{C}$ in a humidified atmosphere containing $5 \% \mathrm{CO}_{2}$.

\subsection{Cell Transfection}

Cells $\left(1.4 \sim 1.6 \times 10^{5}\right.$ cells/well $)$ were seeded in 6-well plates and incubated at $37^{\circ}$ Cuntilthey reached60-70\%confluency. Before transfection, culture medium was removed and replaced with fresh serum-free Opti-MEMI (Gibco) medium. Tumor cells were transfected with siRNA-1 or controls using RNAiMAX (Invitrogen, Grand Island, NY). The cul- 
ture supernatant was replaced after $4 \mathrm{~h}$ and fresh medium containing 10\% FBS was added. The cells were harvested and evaluated after $48 \mathrm{~h}$.

\subsection{Real-time RT-PCR for determination of survivin mRNA}

Survivin gene expression was determined by real-time RT-PCR. Total RNA was extracted from the transfected cells using TRIZOL reagent (TaKaRa, Dalian, China). Complementary deoxyribonucleic acids (cDNAs) were synthesized by reverse transcription from $1 \mu \mathrm{g}$ of total RNA. Forward and reverse primers used were as follows: 5'-CAGTGTTTCTTCTGC TTCAAGG-3' and 5'-CTTATTGTTGGTTTCCTTT GCAT-3' (Sangon Biotech, China). SYBR Green PCR kit (TaKaRa, Dalian, China) was used for the PCR reaction and each sample was analyzed in triplicate. The relative expression of each mRNA was detected and normalized to that of glyceraldehyde-3-phosphate dehydrogenase (GAPDH) mRNA as described [32].

\subsection{Western blot analysis for expression of survivin protein}

Western blot analysis was performed following standard methods [33]. After a period of incubation, cells were washed twice with PBS. Total protein was extracted on ice by RIPA (Sigma-Aldrich, St. Louis, $\mathrm{MO})$. Protein concentration was measured using a bicinchoninic acid (BCA) protein assay kit (Bio-rad, Hercules, CA). Electrophoretic analysis of $30 \mu 1$ protein from each sample was performed by SDS-PAGE. The proteins are transferred to a PVDF membrane after the separation. The detection of target protein was carried out using a secondary antibody (sheep anti-rabbit antibody, 1:10000 dilutions, Pierce, Rockford, IL, USA) following the primary antibody (rabbit anti-human survivin antibody, 1:1000 dilutions, Cell Signaling Technology, Inc.). Binding was detected using an enhanced chemiluminescence (ECL) kit (GE Healthcare, United Kingdom). The results were analyzed by gel imaging and analysis system (Upland, CA, USA).

\subsection{Visualization of survivin expression by fluorescence microscopy}

The expression of survivin in cancer cells was detected using immunofluorescence [34]. Coverslips were placed into a 12 well plate and cells were seeded $\left(1 \times 10^{4}\right)$ into the coverslips/well. The cells were incubated overnight at $37^{\circ} \mathrm{C}$. Tumor cells were transfected with different concentrations of siRNA-1. After 48h, medium was removed from wells. The cells were washed with warm PBS and fixed in $4 \%$ paraformaldehyde/PBS for $15 \mathrm{~min}$. Cells were permeabilized by
TritonX-100 and then treated with primary survivin antibody and FITC-conjugated donkey anti-goat antibody. Then, mounting solution was added. The cells were visualized on a fluorescence microscope in the dark.

\subsection{Flow Cytometry analysis of cell cycle and apoptosis}

Cell cycle and apoptosis were determined by flow cytometry [35]. Cells $\left(2 \times 10^{5}\right.$ cells/well $)$ were plated onto 6-well plates overnight and then replaced with serum free medium for cell cycle synchronization. Then the cells were treated with 10, 20, 40nM siRNA complex.

The cells were harvested and fixed in 75\% methanol at $4^{\circ} \mathrm{C}$ overnight. The cells were washed twice with cold PBS and stained with PI $(0.5 \mathrm{mg} / \mathrm{ml}$ RNase, and $0.1 \mathrm{mg} / \mathrm{ml}$ PI in PBS) for $30 \mathrm{~min}$ at room temperature. The stained cells were characterized by a flow cytometer flow cytometer (Beckman Coulter Corp., Tokyo, Japan), and DNA content was analyzed by FlowJo software.

After $48 \mathrm{~h}$, the cells were harvested and washed twice with PBS. The cells were stained using Annexin V-FITC/PI kit (KeyGEN Biotech, China), following manufacturer's protocol.

After incubation for $20 \mathrm{~min}$ at room temperature in the dark, cell apoptosis was immediately detected on a flow cytometer as described $[36,37]$.

\subsection{MTT Assay}

Cell viability was measured by MTT assay [38]. Cells $\left(1 \times 10^{4}\right.$ cells/well $)$ were grown in 96 -well plates and incubated overnight. Various concentrations of siRNA (0-50nM) and RNAiMAX complexes were added to the cells after the replacement of culture medium with serum free medium. The medium was removed after $4 \mathrm{~h}$ and the cells were cultured in fresh medium for a period of time. MTT assay was per-

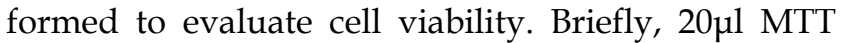
stock solution $(5 \mathrm{mg} / \mathrm{ml}$ in PBS) was added and incubated for $4 \mathrm{~h}$ at $37^{\circ} \mathrm{C}$. The medium was then removed. After that, $150 \mu 1$ / well DMSO was added to dissolve formazan crystals that formed and the absorbance was measured at $540 \mathrm{~nm}$ using an automatic microplate reader (Biotek, VT, USA) [39].

\subsection{Cell proliferation}

Cells $\left(1 \times 10^{4}\right.$ cells/well $)$ were grown in 24 -well plates and incubated overnight. Different concentrations of siRNA complexes were added and after $4 \mathrm{~h}$ incubation, the cells were cultured in fresh medium. Cells from three wells were counted with trypan blue staining method in each group every day. The survival rate of the cells was observed for a total of seven days. 


\subsection{Mitochondrial membrane potential anal- ysis}

JC-1 (5, 5', 6, 6'-tetrachloro-1, 1', 3, 3'-tetraethylbenzimidazol carbocyanine iodide, Sigma-Aldrich), a fluorescent probe, was used to measure alterations in $\Delta \psi \mathrm{m}$. Healthy cells with a high $\Delta \psi \mathrm{m}$ exhibit red fluorescence. Meanwhile, apoptotic or unhealthy cells with a low $\Delta \psi \mathrm{m}$ exhibit green fluorescence (19). HeLa cells were seeded onto dishes at a density of $1 \times 10^{5}$ cells/well. Subsequent to pretreatment with different concentrations of siRNA, cells were incubated with JC- 1 at a final concentration of 2 $\mu \mathrm{M}$ at $37^{\circ} \mathrm{C}$ for $15 \mathrm{~min}$ in the dark. Cells were washed with PBS and changes in mitochondrial fluorescence were analyzed on a fluorescent microscope.

\subsection{Statistical Analysis}

The data were analyzed using SPSS 16.0 software (IBM, Armonk, New York). The difference between two independent samples was analyzed by Student's $\mathrm{t}$ test. A statistically significant difference was considered to be present at $p<0.05$.

\section{Conclusions}

Because survivin is an essential component of most types of cancers [40-42], it can serve as a biomarker for a number of malignancies [43-45]. Although targeting survivin by siRNA has shown some promise in cancer, the potency of siRNA needs further improvement. In order to achieve maximum therapeutic effect, however, it is essential to identify the most active form of siRNA drugs. In this respect, rational design of the siRNA itself must precede the design of the delivery vehicle.

We have shown that a novel siRNA sequence we designed (siRNA-1), which was modified by several 2'-OMe chemical groups in both strands, is much better than the positive control sequence reported previously. In addition, the suppression of siRNA was cell-line dependent. Meanwhile, siRNA-1 exerted a significant effect on cell proliferation and cell cycle by enhancing the G2/M phase arrest. The contribution of siRNA to cell proliferation, inhibition, and cell apoptosis were also regulated and controlled by signaling pathways. For instance, Bad and Bax expression increased in the cancer cells treated with siRNA, along with induction of apoptosis. Moreover, the Akt-mediated signal pathway may be involved in apoptosis induction. In response to apoptotic stimuli, survivin is trafficked from the mitochondria to the cytosol where it can inhibit apoptosis. Similarly the decreasing $\Delta \psi \mathrm{m}$ is one of the important hallmarks of cellular apoptosis $[46,47]$. The results of this study suggest that the novel siRNA sequence has high potency and warrants further evaluation as a therapeutic agent. Clinical translation of siRNA requires both optimization of the siRNA design and development of an efficient delivery vehicle. Our future work will focus on assessing the in vivo activities of the novel survivin siRNA.

\section{Author Contributions}

Yuhuan Li and Da Liu performed the laboratory experiments. Yuhuan $\mathrm{Li}$ drafted the manuscript; Lesheng Teng and Robert. J Lee conceived and designed most of the studies and revised the manuscript; Yong Cai and Jing Xie participated in study design and the manuscript revision; Yulin Zhou and Yujing $\mathrm{Li}$ contributed research materials and were involved in helpful discussions.

\section{Competing Interests}

The authors have declared that no competing interest exists.

\section{References}

1. Srinivasula SM, Ashwell JD. IAPs: what's in a name? Mol Cell. 2008; 30: 123-35.

2. Dohi T, Okada K, Xia F, et al. An IAP-IAP complex inhibits apoptosis. J Biol Chem. 2004; 279: 34087-90.

3. Mita AC, Mita MM, Nawrocki ST, et al. Survivin: Key regulator of mitosis and apoptosis and novel target for cancer therapeutics. Clin Cancer Res. 2008; 14: 5000-5.

4. Liu T, Biddle D, Hanks AN, et al. Activation of dual apoptotic pathways in human melanocytes and protection by survivin. J Invest Dermatol. 2006; 126: 2247-56.

5. Colnaghi R, Wheatley SP. Liaisons between Survivin and Plk1 during Cell Division and Cell Death. J Biol Chem. 2010; 285: 22592-604.

6. Zang XP, Pento JT. SiRNA inhibition of ER-alpha expression reduces KGF-induced proliferation of breast cancer cells. Anticancer Res. 2008; 28: 2733-5.

7. Waligorska-Stachura J, Andrusiewicz M, Sawicka-Gutaj N, et al. Survivin Delta Ex3 Overexpression in Thyroid Malignancies. Plos One. 2014; 9.

8. Zeng W, Meng FK, Liu ZM, et al. Bortezomib-based chemotherapy regimens can improve response in newly diagnosed multiple myeloma patients with bcl-2 and survivin overexpression. Int J Clin Exp Patho. 2014; 7: 4239-46.

9. Poomsawat S, Punyasingh J, Vejchapipat P. Overexpression of Survivin and Caspase 3 in Oral Carcinogenesis. Appl Immunohisto M M. 2014; 22: 65-71.

10. Tazo Y, Hara A, Onda T, et al. Bifunctional roles of survivin-Delta Ex3 and survivin-2B for susceptibility to apoptosis in endometrial carcinomas. J Cancer Res Clin. 2014; 140: 2027-37.

11. Liu $\mathrm{YB}, \mathrm{Gao} \mathrm{XH}$, Deeb $\mathrm{D}$, et al. Ubiquitin-proteasomal degradation of antiapoptotic survivin facilitates induction of apoptosis in prostate cancer cells by pristimerin. Int J Oncol. 2014; 45: 1735-41.

12. Dohi T, Beltrami E, Wall NR, et al. Mitochondrial survivin inhibits apoptosis and promotes tumorigenesis. J Clin Invest. 2004; 114: 1117-27.

13. Altieri DC. Survivin and IAP proteins in cell-death mechanisms. Biochem J. 2010; 430: 199-205.

14. Talbot DC, Blackhall FH, Kowalski D, et al. A randomized open-label phase II study evaluating antitumor activity of the survivin antisense oligonucleotide LY2181308 (LY) in combination with docetaxel (DO) for second-line treatment of patients with non-small cell lung cancer (NSCLC) using change in tumor size (CTS). J Clin Oncol. 2013; 31.

15. Mobahat M, Narendran A, Riabowol K. Survivin as a Preferential Target for Cancer Therapy. Int J Mol Sci. 2014; 15: 2494-516.

16. Hendruschk S, Wiedemuth R, Aigner A, et al. RNA interference targeting survivin exerts antitumoral effects in vitro and in established glioma xenografts in vivo. Neuro Oncol. 2011; 13: 1074-89.

17. Yang ZG, Yu B, Zhu J, et al. A microfluidic method to synthesize transferrin-lipid nanoparticles loaded with siRNA LOR-1284 for therapy of acute myeloid leukemia. Nanoscale. 2014; 6: 9742-51.

18. Boukany PE, Morss A, Liao WC, et al. Nanochannel electroporation delivers precise amounts of biomolecules into living cells. Nat Nanotechnol. 2011; 6: 747-54.

19. Wen Y, Meng WS. Recent In Vivo Evidences of Particle-Based Delivery of Small-Interfering RNA (siRNA) into Solid Tumors. J Pharm Innov. 2014; 9 : 158-73. 
20. Zhou CG, Yang ZG, Teng LS. Nanomedicine based on Nucleic Acids: Pharmacokinetic and Pharmacodynamic Perspectives. Curr Pharm Biotechno. 2014; 15: 829-38.

21. Yu B, Wang XM, Zhou CG, et al. Insight into Mechanisms of Cellular Uptake of Lipid Nanoparticles and Intracellular Release of Small RNAs. Pharm Res-Dordr. 2014; 31: 2685-95.

22. Elbashir SM, Harborth J, Lendeckel W, et al. Duplexes of 21-nucleotide RNAs mediate RNA interference in cultured mammalian cells. Nature. 2001; 411: 494-8.

23. Devi GR. siRNA-based approaches in cancer therapy. Cancer Gene Ther. 2006; 13: 819-29.

24. Seth S, Matsui Y, Fosnaugh K, et al. RNAi-based Therapeutics Targeting Survivin and PLK1 for Treatment of Bladder Cancer. Mol Ther. 2011; 19: 928-35.

25. Li XJ, Zhang XY, Li XY, et al. The Role of Survivin in Podocyte Injury Induced by Puromycin Aminonucleoside. Int J Mol Sci. 2014; 15: 6657-73.

26. Li SD, Huang L. Targeted delivery of antisense oligodeoxynucleotide and small interference RNA into lung cancer cells. Mol Pharmaceut. 2006; 3: 579-88.

27. Lee SJ, Son S, Yhee JY, et al. Structural modification of siRNA for efficient gene silencing. Biotechnol Adv. 2013; 31: 491-503.

28. Prakash TP, Allerson CR, Dande P, et al. Positional effect of chemical modifications on short interference RNA activity in mammalian cells. J Med Chem. 2005; 48: 4247-53.

29. Kraynack BA, Baker BF. Small interfering RNAs containing full 2 '-O-methylribonucleotide-modified sense strands display argonaute2/eIF2C2-dependent activity. Rna. 2006; 12: 163-76.

30. Castanotto D, Rossi JJ. The promises and pitfalls of RNA-interference-based therapeutics. Nature. 2009; 457: 426-33.

31. Zheng J, Zhang L, Zhang J, et al. Single modification at position 14 of siRNA strand abolishes its gene-silencing activity by decreasing both RISC loading and target degradation. FASEB J. 2013; 27: 4017-26.

32. Chakrabarti S, Wu XF, Yang ZG, et al. MOG1 Rescues Defective Trafficking of $\mathrm{Na}(\mathrm{v}) 1.5$ Mutations in Brugada Syndrome and Sick Sinus Syndrome. Circ-Arrhythmia Elec. 2013; 6: 392-401.

33. Yang ZG, Sun $W, H u$ KL. Adenosine A(1) receptors selectively target protein kinase $\mathrm{C}$ isoforms to the caveolin-rich plasma membrane in cardiac myocytes. Bba-Mol Cell Res. 2009; 1793: 1868-75.

34. Yang ZG, Sun W, Hu KL. Molecular mechanism underlying adenosine receptor-mediated mitochondrial targeting of protein kinase C. Bba-Mol Cell Res. 2012; 1823: 950-8.

35. Zhang YP, Kong QH, Huang $\mathrm{Y}$, et al. Inhibition of c-FLIP by RNAi enhances sensitivity of the human osteogenic sarcoma cell line U2OS to TRAIL-induced apoptosis. APJCP. 2015; 16: 2251-6.

36. Xie J, Teng LS, Yang ZG, et al. A Polyethylenimine-Linoleic Acid Conjugate for Antisense Oligonucleotide Delivery. Biomed Res Int. 2013.

37. Jin LL, Batra S, Douda DN, et al. CXCL1 Contributes to Host Defense in Polymicrobial Sepsis via Modulating T Cell and Neutrophil Functions. J Immunol. 2014; 193: 3549-58.

38. Schroeder BR, Ghare MI, Bhattacharya C, et al. The disaccharide moiety of bleomycin facilitates uptake by cancer cells. J Am Chem Soc. 2014; 136: 13641-56.

39. Wang XM, Huang XM, Yang ZG, et al. Targeted Delivery of Tumor Suppressor microRNA-1 by Transferrin- Conjugated Lipopolyplex Nanoparticles to Patient-Derived Glioblastoma Stem Cells. Curr Pharm Biotechno. 2014; 15: 839-46.

40. Xing Z, Conway EM, Kang C, et al. Essential role of survivin, an inhibitor of apoptosis protein, in T cell development, maturation, and homeostasis. J Exp Med. 2004; 199: 69-80.

41. Roca H, Varsos ZS, Mizutani K, et al. CCL2, survivin and autophagy: new links with implications in human cancer. Autophagy. 2008; 4: 969-71.

42. Small S, Keerthivasan G, Huang Z, et al. Overexpression of survivin initiates hematologic malignancies in vivo. Leukemia. 2010; 24: 1920-6.

43. Ikeguchi M, Kaibara N. survivin messenger RNA expression is a good prognostic biomarker for oesophageal carcinoma. Br J Cancer. 2002; 87: 883-7.

44. Lu B, Gonzalez A, Massion PP, et al. Nuclear survivin as a biomarker for non-small-cell lung cancer. British journal of cancer. 2004; 91: 537-40.

45. Brennan DJ, Rexhepaj E, O'Brien SL, et al. Altered cytoplasmic-to-nuclear ratio of survivin is a prognostic indicator in breast cancer. Clin Cancer Res. 2008; 14: 2681-9.

46. Dohi T, Beltrami E, Wall NR, et al. Mitochondrial survivin inhibits apoptosis and promotes tumorigenesis. J Clin Invest. 2004; 114: 1117-27.

47. Chen S, Liu J, Liu X, et al. Panax notoginseng saponins inhibit ischemia-induced apoptosis by activating PI3K/Akt pathway in cardiomyocytes. J Ethnopharmacol. 2011; 137: 263-70. 